\title{
ON THE EQUATION
}

$$
a\left(x^{m}-1\right) /(x-1)=b\left(y^{n}-1\right) /(y-1)
$$

$$
\text { By }
$$

T. N. SHOREY

\& 1. Let $\mathrm{m}>1, \mathrm{v}>1, \mathrm{x}>1, \mathrm{y}>1$ and $\mathrm{a}, \mathrm{b}$ with $(\mathrm{a}, \mathrm{b})=1$ be positive integers satisfying $a(y-1) \neq b(x-1)$. This equation of Goormaghtigh arose from the question whether an integer has all the digits identically equal in their expansions to two distinct bases. It follows from Baker's effective version [1] of Thue's theorem [6] that the equation

$$
a^{\frac{m}{x^{2}}-1}=b^{\frac{y^{n}-1}{y}-1}
$$

implies that $\max (\mathrm{n}, \mathrm{n})$ is bounded by an effectively computable number depending only on $a, b, x$ and $y$. Further Balasubramanian and the author [3] applied the theory of linear forms in logarithms to generalise this result by showing that equation ( 1 implies that $\max (a, b, x, y, m, n)$ is bounded by an effectively computable number depending only on the greatest prime factor of abxy. In this paper, we apply the theory of linear forms in logarithms to obtain the following generalisations. We shall always write $z-\max (x, y)$.

\section{Theorem 1 .}

Let $0<v<1$ and $\mathrm{F}>1$. If positive integers $\mathrm{m}>\mathrm{a}>1$; $x>1, y>1, a$ and $b$ with $a<x, b<y, a(y-1) \neq b(x-1)$ and

$$
|x-y| \leqslant \max \left(1,2^{6}\right)
$$


satisfy (1), then $\mathrm{m}$ is bounded by an effectively computable number depending only on $\theta$ and $\mathrm{F}$.

For an account of earlier result in the direction of equation (1), see [3]. We combine theorem 1 with an elementary argument to obtain the following result.

\section{Theorem 2.}

Let $\mathrm{F}_{1}>1$. There exists an effectively computable absolute constant $\mathrm{C}>0$ and an effectively computable number $\varepsilon_{1}>0$ depending only on $F_{1}$ such that equation (1) in positive integers $\mathrm{m}>\mathrm{n}>1, \mathrm{~m}>2, \mathrm{x}>1, \mathrm{y}>1$, a and $\mathrm{b}$ with $(\mathrm{a}, \mathrm{b})=1, \mathrm{a}, \mathrm{x}, \mathrm{b}<\mathrm{y}, \mathrm{a}(\mathrm{y}-1) \neq \mathrm{b}(\mathrm{x}-1)$ and

$$
|x-y|<\max \left(F_{i},(\log z)^{C}\right)
$$

implies that

$$
\max (m, n, x, y, a, b)<c_{i}
$$

Combining theorem 1 with lemma 1 and an estimate on p-adic linear forms in logarithms, we have

Theorem 3.

If $\mathrm{m}>1, \mathrm{n}>1, \mathrm{x}>1$ and $\mathrm{y}>1$ with $(\mathrm{x}, \mathrm{y})=1$ satisfy

$$
\frac{x^{m}-1}{x-1}=\frac{y^{n}-1}{y-1}
$$

then

$$
\max (\mathrm{m}, \mathrm{n}, \mathrm{x}, \mathrm{y})<\mathrm{C}_{2}
$$

where $G_{2}>0$ is an effectively computable number depending only on the greatest prime factor of $\mathrm{x}(\mathrm{y}-\mathrm{x})$. 
If $\mathrm{a}$ and $\mathrm{b}$ are fixed, the restriction (3) in theorem 2 can be reiaxed considerably.

\section{Theorem 4.}

Let $\mathrm{a}$ and $\mathrm{b}$ be positive integers. Let $\mathrm{F}_{2}>1$. There exists an effectively computable number $\mathbf{C}_{3}>0$ depending only on $\mathrm{a}, \mathrm{b}$ and $\mathrm{F}_{2}$ such that equation (1) in positive intogers $\mathrm{m}>\mathrm{n}>1$, $\mathrm{x}>1, \mathrm{y}>1$ with $\mathrm{a}(\mathrm{y}-1) \not \neq \mathrm{b}(\mathrm{x}-1)$ and

$$
|x-y|<F_{2} z /(\log z)^{2}(\log \log z)^{3}
$$

implies that

$$
\max (m, n, x, y)<C_{3} .
$$

12. The proof of these results depend on the following application of a theorem of Baker [2] on linear forms in logarithms.

\section{Lemma 1 .}

Let $\mathrm{F}_{3}>1$. Let $\mathrm{m}, \mathrm{n}, \mathrm{x}>1, \mathrm{y}>1, \mathrm{a}<\mathrm{F}_{3} \mathrm{x}$, and $\mathrm{b}<\mathrm{F}_{3} \mathrm{y}$ with $\mathrm{a}(\mathrm{y}-1) \neq \mathrm{b}(\mathrm{x}-1)$ satisfy (1). Put $\mathrm{z}=\max (\mathrm{x}, \mathrm{y})$ and $z_{1}=\min (x, y)$.

Then

$$
\max (m, n) \leqslant C_{4}(\log z)^{3}(\log \log z)^{2} /\left(\log z_{1}\right)
$$

where $\mathrm{C}_{4}>0$ is an effectively computable number depending only on $\mathrm{F}_{3}$.

\section{Proof of Lemma 1.}

We may assume that $i n>n$. Denote by $C_{1}>0$ and $\boldsymbol{E}_{2}>0$ effectively computable numbers depending only on $\boldsymbol{F}_{3}$. 
By equation (1), we have

$$
0 \neq\left|\frac{a x^{m}}{x-1}-\frac{b y^{n}}{y-1}\right|<C_{1}
$$

By: an estimate of Baker [2], the left hand side of inequality (6) exceeds $x^{m} \exp \left(-C_{2}(\log m)(\log z)^{3}(\log \log z)\right)$.

Now the lemma follows immediately by combining these estimates.

\section{Proof of theorem 1.}

Denote by $\mathrm{C}_{3}, \mathrm{C}_{4}, \ldots, \mathrm{C}_{9}$ effectively computable positive numbers depending only on $\theta$ and F. Suppose that the assumptions of theorem $I$ are satisfied. Then, by lemma 1 and (2), we have

$$
\mathrm{m} \leq \mathrm{C}_{3}(\log \mathrm{x})^{3} .
$$

In view of (7) and (2), we may assume that

$$
\min (x, y)>C_{4}
$$

with $\mathrm{C}_{4}$ sufficiently large. Further, by (1), we have

$$
\begin{aligned}
& a x^{m-1}<b y^{n-1}\left(1+\frac{2}{y}\right), \\
& h y^{n-1}<a x^{m-1}\left(1+\frac{2}{x}\right)
\end{aligned}
$$

Now it follows from (9), (2), (7) and (8) that either $m=a$ or $\mathrm{m}=\mathrm{n}+1$.

Let $m=n$. It follows from (9), (i) and (7) that

$$
\left|\log \frac{a}{b}\right|<C_{5} x^{-1+\theta}(\log x)^{3} \text {. }
$$


Further, by (2!, we have

$$
\max \left(\left|\log \frac{x}{y}\right|,\left|\log \left(\frac{x-1}{y-1}\right)\right|\right)<c_{6} x^{-1+\theta}
$$

Consequently

$$
\left|\log \frac{a(y-1)}{b(x-1)}\right|<\left(C_{5}+C_{6}\right) x^{-1+\theta}(\log x)^{3}
$$

Now it follows from an estimate of Waldschmidt [7] or Ramachandra and the author [5] (in the latter reference, the arguments allow to prove the estimate without the restriction on the multiplicative independence of $\alpha_{1}$ and $\alpha_{2}$ ) on linear forms in logarithms that the left hand side of inequality (6), with $\mathrm{m}=\mathrm{n}$ and $\mathrm{F}_{3}=1$, exceeds $\mathrm{x}^{\mathrm{m}-\mathrm{C}_{7}}$.

Thus, by (6), $x^{m-C_{7}}<C_{1}$ which implies that $m \leq C_{8}$, since $x>1$.

Let $m=n+1$. Re-write equation (1) as

$$
a x \frac{x^{n}-1}{x-1}=b \frac{y^{n}-1}{y-1}-a \text {. }
$$

Now argue as in the case $m=n$ to conclude that $m<C_{9}$. This completes the proof of theorem 1 .

Proof of theorem 2.

We shall choose, later, an effectively computable absolute constant $\mathrm{C}$ satisfying $0<\mathrm{C}<1$.

Case I :

$$
F_{1}=\log _{z}{ }^{C} \text {. Then } \mid x-y: \leq F_{1} \text {. Hen.e, hy theorem }
$$

1. we see that $m$ is bounded by an effectively computable 
number depending only on $F_{1}$. Now the assertion of theorem 2 follows from (1), since $(a, b)=1$.

Case 1I:

$$
\begin{aligned}
& F_{1}<(\log z)^{C} \text {. Then, by (3), observe that } \\
& |x-y|<(\log z) C \log z<\max \left(27, z^{\frac{1}{3}}\right) .
\end{aligned}
$$

Now apply theorem 1 to conslude that $m$ is bounded by an effectively computable absolute constant $\mathrm{C}_{10}$. Let $2<\mathrm{m}<\mathrm{C}_{10}$ be given. Denote by $\mathrm{C}_{11}, \mathrm{C}_{12}, \ldots \ldots, \mathrm{C}_{16}$ effectively computable positive numbars depzndilag only on $\mathrm{m}$. We may assume that $\min (x, y)>C_{11}$ with $C_{11}$ sufficiently large. otherwise the assertion of theorem 2 follows from (1) and $(a, b)=1$. Then equation (1) implies that $m=n$ or $m=n+1$.

If $x=y$, then $a \neq b$ and equation (1) implies that

$$
\mathrm{x}^{\mathrm{n}}\left(\mathrm{ax}^{\mathrm{m}-\mathrm{a}}-\mathrm{b}\right)=\mathrm{a}-\mathrm{b}
$$

which, since $n>1$, is not possible if $C_{11}$ is sufficiently large. Thus we may assume that $x \neq y$.

Re-write equation (1) as

$$
a\left(\frac{P_{m}(x)}{d}\right)=b\left(\frac{P_{n}(y)}{d}\right)
$$

where

$$
P_{m}(X)=\frac{X^{m}-1}{X-1}, \quad P_{n}(Y)=\frac{Y^{n}-1}{Y-1}
$$

and $d$ is the greatest common divisor of $P_{m}(x)$ and $P_{n}(y)$. Thus

$$
P_{m}(x) d^{-1}<b<2 x 。
$$


Put

$$
P_{m}=e^{2 \pi i / m}, P_{n}=e^{2 \pi i / n}, K=Q\left(P_{m}, P_{n}\right)
$$

For a prime $\mathrm{p}$ dividing $\mathrm{d}$, let ord ${ }_{\mathrm{p}}(\mathrm{d})=\alpha_{\mathrm{p}}$. Let $\wp$ be a prime ideal in the ring of integers of $K$ dividing $p$. Then $\delta$ divides an ideal

$$
\left[x-y-p_{m}^{r}+P_{n}^{s}\right]
$$

for some positive integers $\mathrm{r}<\mathrm{m}$ and $\mathrm{s}<\mathrm{n}$.

Put

$$
\sigma=e^{2 \pi \mathrm{i} / 6}, \mathrm{~T}_{1}=\left\{\sigma, \sigma^{5}\right\}, \mathrm{T}_{2}=\left\{\sigma^{2}, \sigma^{4}\right\} .
$$

Suppose that (11) is a zero ideal. Then, since $x \neq y$ and $1<\mathrm{r}<\mathrm{m}, \quad \mathrm{J}<\mathrm{s}<\mathrm{n}$, we see that $|\mathrm{x}-\mathrm{y}|=1$. Then $\cos \left(\frac{2 \pi r}{m}\right)= \pm 1+\cos \left(\frac{2 \pi s}{n}\right), \sin \left(\frac{2 \pi r}{m}\right)=\sin \left(\frac{2 \pi s}{n}\right)$.

These equations imply that either $\rho_{m}^{r} \varepsilon T_{1}, P_{n}^{s} \varepsilon T_{2}$ or $P_{m}^{r} \in T_{2}, P_{n}^{s} \in T_{1}$. Thus $m \neq n+1$, since $m$ and $n$ are divisible by 3 . Therefore $m=n$. Then $m$ is even, since $m$ is divisible by 6 . If $x-y=1$, then equation (1) with $m=n$ implies that $\mathrm{x}=\mathrm{y}+1$ divides $\mathrm{a}\left(\mathrm{x}^{\mathrm{m}-1}+\ldots+1\right)$. Therefore $\mathrm{x}$ divides $a$, which is not possible, since $a<x$. Similarly if $y-x=1$ and $m$ even, equation (1) with $m=n$ has no solution. Thus we may assume that $(11)$ is a non-zero ideal.

Put

$$
\mathrm{C}^{\prime}=\left(2 \mathrm{~m}^{2}\right)^{-1}
$$

Then, since $\oint_{0}$ divides a non-zero ideal (11). we obtain by teking norms, 


$$
p<C_{12}(\log x)^{t} \text {. }
$$

Infact either $a_{\mathrm{p}}<\mathrm{C}_{13}$ or $\rho^{a} \mathrm{p}^{\mathrm{p}_{13}}$. divides a non-zero ideal of the form (11). Therefore

$$
{ }_{\mathrm{p}}^{\mathrm{a}}<(\log \mathrm{x}){ }^{\mathrm{C}_{14}}
$$

Hence we conclude $q<x^{\frac{1}{4}}$ which, together with (10), implies that

$$
\mathrm{x}^{\mathrm{m}-1}<\mathrm{P}_{\mathrm{m}}(\mathrm{x})<2 \mathrm{x}^{3 / 2} .
$$

Then, since $m>2$, we conclude that $x \leqslant C_{15}$. Then $y<2 x<2 C_{15}$. Further, by (1) and $(a, b)=1$, we see that $\max (a, b) \leqslant C_{16}$. This completes the proof of theorem 2 .

\section{Proof of Theorem 3.}

We may assume that $m \neq n$, otherwise equation (4) has no solution, since $\mathrm{x} \neq \mathrm{y}$. Denote by $\mathrm{C}_{17}, \mathrm{C}_{18}, \ldots, \mathrm{C}_{22}$ effectively computable positlve numbers depending only on the greatest prime factor of $x(y-x)$. Put $y-x=k$. Then it follows from equation (4) and $(x, y)=1$ that $k$ divides

$$
\left(x^{|m-n|}-1\right) /(x-1) \text {. }
$$

Thus, for a prime p dividing $\mathrm{k}$, we have

$$
\operatorname{ord}_{p}(k)<\operatorname{ord}_{p}\left(x^{|m-n|}-1\right) \text {. }
$$

Now we apply an estimate of van der Poorten [4] on p-adic linear forms in logarithms to obtain

$$
\operatorname{ord}_{p}(k)<C_{17}(\log i m+n i+\log \log x)^{2}
$$


Thus

$$
\log |\mathrm{k}|<\mathrm{C}_{18}(\log |\mathrm{m}+\mathrm{n}|+\log \log x)^{2} .
$$

By lemma 1, we have

$$
\max (m, n) \leqslant C_{19}(\log z)^{4} .
$$

Therefore

$$
\log |\mathrm{k}| \leqslant \mathrm{C}_{20}\left(\log \log \mathrm{z}^{2} .\right.
$$

Now we apply theorem 1 to sonclude that $\max (m, n) \leqslant \mathrm{C}_{21}$. If $\max (x, y)>C_{22}$ with $C_{22}$ sufficiently large, then it follows from (12) and (4) that $m=n$. This is not possible, since $x \neq y$. This completes the proof of theorem 3 .

\section{Proof of theorem 4.}

Suppose that the assumptions of theorem 4 are satisfied. Denote by $\mathrm{C}_{23}, \mathrm{C}_{24}$, ... effectively computable positive numbers depending only on $a, b$ and $F_{2}, \quad$ By lemma 1 and (5) we have

$$
m \leqslant C_{23}((\log x)(\log \log x))^{2} \text {. }
$$

In view of (13) and (5), we may assume that $\max (x, y)>C_{24}$ with $\mathrm{C}_{24}$ sufficiently large. Then we use inequality (9) to conclude that equation (1) implies that $m=n$. Therefore we may assume that $a \neq b$, otherwise $x \neq y$ and equation (1) with $m=n$ has no solution. Then, by again applying (9), we see from (5) and (13) that

$$
\mathrm{c}_{25}<\left|\log \frac{\mathrm{a}}{\mathrm{b}}\right|<\mathrm{c}_{26}(\log \log \mathrm{x})^{-1}
$$

which implies that $x<C_{27}$. Hence, by (5) and (13), we conclude that $\max (\mathrm{m}, \mathrm{x}, \mathrm{y})<\mathrm{C}_{28}$. This completes the proof of theorem 4 . 


\section{References}

1. A. Baker, Contributions to the theory of Diophantine equations 1: On the representation of integers by binary forms, Phil. Trans. Roy. Soc. London, A 263 (1968), 173-208.

2. A. Baker, The theory of linear forms in logarithms, Transcendence theory: Advances and applications, Academic Press, London and New York (1977), 1-27.

3. R. Balasubramanian and T. N. Shorey, On the equation $a\left(x^{m}-1\right) /(x-1)=b\left(y^{n}-1\right) /(y-1)$, Math. Scand. 46 (1980), 177-182.

4. A. J. Van der Poorten, Linear forms in logarithms in the p-adic case, Transcendence theory: Advances and applications, Academic Press, London and New York (1977), 29-57.

5. K. Ramachandra and T. N. Shorey, On gaps between numbers with a large prime factor, Acta Arlth. 24 (1973), 99-111.

6. A. Thue, Über Annaherungswerte algebraischer Zahlen, J. reine angew. Math. 135 (1909), 284-305.

7. M. Waldschmidt, $A$ lower bound for linear forms in logarithms, Acta Arith. 37 (1980), 257-283.

School of Mathematics

Tata Institute of Fundamental Research

Homi Bhabha Road

Bombay 400005 (India) 